\title{
Numerical study of threshold gain behavior for a THz random laser in a two-dimensional active disordered medium with a three-level atomic system
}

\author{
LIU Yong, LIU JinSong ${ }^{*} \&$ WANG KeJia \\ Wuhan National Laboratory for Optoelectronics, School of Optoelectronic Science and Engineering, Huazhong University of Science and Tech- \\ nology, Wuhan 430074, China
}

Received March 17, 2011; accepted May 3, 2011

\begin{abstract}
By numerically solving Maxwell's equations and rate equations in a two-dimensional (2D) active random media made of ruby grains with a three-level atomic system, the threshold gain behavior for a $\mathrm{THz}$ random laser is investigated. The spectral intensity variation with the pumping rate is calculated for both the transverse magnetic (TM) field and the transverse electric (TE) field. The computed results show that THz random lasing could occur in a 2D disordered medium for both the TM and TE cases. Further analysis reveals that the THz lasing threshold for TM fields is lower than that for TE fields.
\end{abstract}

random lasers, polarization, terahertz

Citation: Liu Y, Liu J S, Wang K J. Numerical study of threshold gain behavior for a THz random laser in a two-dimensional active disordered medium with a three-level atomic system. Chinese Sci Bull, 2011, 56: 2664-2667, doi: 10.1007/s11434-011-4633-6

Since random laser action was first predicted by Letokhov in 1968 [1] and observed by Lawandy in 1994 [2], many experimental and theoretical studies have been performed [3-17]. To numerically simulate the propagation and localization of electromagnetic waves in such random media, the finite-difference time-domain (FDTD) method has been widely used. Moreover, various types of auxiliary differential equation FDTD (ADE-FDTD) approaches for the analysis of nonlinear and active systems have also been developed. Jiang and Soukoulis successfully reproduced random lasing in one-dimensional (1D) random media using a semiclassical model. In this model, FDTD method is coupled with the rate equation in a four-level energy structure [6]. Their methodology has been extended to the twodimensional (2D) case.

Note that the search for efficient, high-power, inexpensive, and compact methods for the generation of coherent $\mathrm{THz}$ radiation has been a primary research thrust in modern optoelectronics and photonics [18-25]. For this, recently, a

*Corresponding author (email: jsliu4508@vip.sina.com)
1D theoretical model was built for active disordered media made of ruby grains with a three-level atomic system. These numerical results revealed that terahertz $(\mathrm{THz})$ random lasing could occur in the 1D case under suitable conditions [17]. Although 1D random laser can reveal qualitative properties in a real random lasing system, there are still many physical mechanisms which cannot be investigated using a 1D model. Therefore, the 2D model has been widely used to research random lasing in the optical band $[6,12-16]$.

We aim to construct a model to reveal $\mathrm{THz}$ random lasing effects in the $2 \mathrm{D}$ case. In this work, by numerically solving Maxwell's equations and rate equations in a 2D active random media made of ruby grains with a three-level atomic system, the threshold gain behavior of a $\mathrm{THz}$ random laser is investigated. Using this theory, numerical calculations are performed. The results show that $\mathrm{THz}$ random lasing could occur in a 2D disordered medium and that the lasing threshold for transverse magnetic (TM) fields is lower than that for transverse electric (TE) fields. We believe that these results will provide some valuable predictions that 
will motivate future experimental observations.

\section{Theoretical model}

Our model starts with a 2D square disordered medium with area, $l^{2}$. It consists of circular particles with radius $r$, optical index $n_{2}$, and surface-filling fraction $\Phi$. This is shown in Figure 1(a). These disordered particles are randomly distributed in a background medium with refractive index $n_{1}$. Using the given medium parameters (i.e. $l, r, \Phi, n_{1}$ and $n_{2}$ ), a wide variety random media can be built. The medium parameters used in the following numerical calculations are: $l$ $=5 \mathrm{~cm}, r=80 \mu \mathrm{m}, \Phi=40 \%, n_{1}=1, n_{2}=10$. Note that the refractive index for $\mathrm{THz}$ radiation in ruby is twice that of the one for visible wavelengths [26].

For a TM field, Maxwell's equations are

$$
\begin{gathered}
\mu_{0} \frac{\partial H_{x}}{\partial t}=-\frac{\partial E_{z}}{\partial y}, \\
\mu_{0} \frac{\partial H_{y}}{\partial t}=\frac{\partial E_{z}}{\partial x}, \\
\varepsilon_{i} \varepsilon_{0} \frac{\partial E_{z}}{\partial t}+\frac{\partial P_{z}}{\partial t}=\frac{\partial H_{y}}{\partial x}-\frac{\partial H_{x}}{\partial y},
\end{gathered}
$$

where $E_{z}$ for TM field is the $z$ component of the electric field: $\vec{E}=E_{x} e_{x}+E_{y} e_{y}+E_{z} e_{z}$. In this case, $e_{x}, e_{y}$ and $e_{z}$ are the unit vectors in the $x, y$ and $z$ directions, respectively. $P_{z}$ is the $z$ component of the polarization density vector: $\vec{P}=P_{x} e_{x}+P_{y} e_{y}+P_{z} e_{z}$. In this case, $P_{x}$ and $P_{y}$ are the polarization density components in the $x$ and $y$ directions, respectively. $n_{i}^{2}$ and $\mu_{0}$ are the electric permittivity and the magnetic permeability in a vacuum, respectively. $\varepsilon_{i}=n_{i}^{2}(i=1,2)$.

If the active medium includes ruby, it can be described as a three-level atomic system. For such a system, the three-level rate equations are [17]

$$
\begin{gathered}
\frac{\mathrm{d} N_{1}}{\mathrm{~d} t}=\frac{N_{2}}{\tau_{21}}+\frac{N_{3}}{\tau_{31}}-N_{1} W_{p}, \\
\frac{\mathrm{d} N_{2}}{\mathrm{~d} t}=\frac{N_{3}}{\tau_{32}}-\frac{N_{2}}{\tau_{21}}-\frac{N_{2}}{T}+\frac{E_{z}}{\hbar \omega_{l}} \cdot \frac{\mathrm{d} P_{z}}{\mathrm{~d} t}, \\
\frac{\mathrm{d} N_{3}}{\mathrm{~d} t}=N_{1} W_{p}-\frac{N_{3}}{\tau_{31}}-\frac{N_{3}}{\tau_{32}}+\frac{N_{2}}{T}-\frac{E_{z}}{\hbar \omega_{l}} \cdot \frac{\mathrm{d} P_{z}}{\mathrm{~d} t} .
\end{gathered}
$$
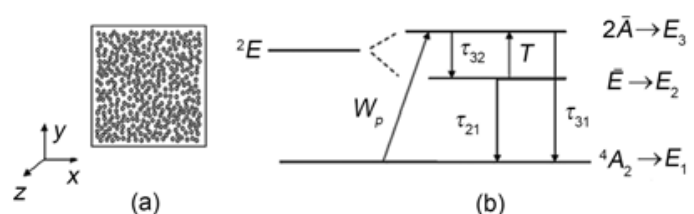

(b)

Figure 1 (a) Schematic diagram of the 2D random medium made of ruby grains; (b) the energy levels for $\mathrm{Cr}^{3+}$ in ruby.
In the above rate equations, the system treated here is a simplified yet realistic three-level atomic system (Figure 1(b)) with energy levels $E_{1}, E_{2}, E_{3}$, and their corresponding populations $N_{1}, N_{2}$ and $N_{3}$, respectively. $W_{p}$ is the rate of external optical pumping at $693.9 \mathrm{~nm}$ that transfers atoms from the ground state $\left(E_{1}\right)$ to level $E_{3} .1 / \tau_{i j}$ is the spontaneous decay rate for an atom in energy level $E_{i}$ to level $E_{j} . T$ is the time of flight between emission and re-absorption for a $29 \mathrm{~cm}^{-1}$ phonon, and it was chosen to be $1 \times 10^{-9} \mathrm{~s}$ in our system [17]. $\omega_{l}=\left(E_{3}-E_{2}\right) / \hbar=2 \pi \times 0.87 \mathrm{THz}$ is the transition frequency from level $E_{3}$ to level $E_{2}$. The term, $\left(E / \hbar \omega_{l}\right) \mathrm{d} P / \mathrm{d} t$ is the classical expression for the instantaneous energy transfer divided by the energy per photon, and is equivalent to the stimulated transition probabilities that are more commonly used in the rate equation above.

The polarization density component $\left(P_{z}\right)$ satisfies the following equation:

$$
\frac{\mathrm{d}^{2} P_{z}}{\mathrm{~d} t^{2}}+\Delta \omega_{l} \frac{\mathrm{d} P_{z}}{\mathrm{~d} t}+\omega_{l}^{2} P_{z}=\kappa \Delta N E_{z}
$$

where $\Delta \omega_{l}=1 / \tau_{32}+2 / T_{2}$ is the actual linewidth (gain spectrum width) of the transition centered at angular frequency $\omega_{l} . T_{2}$ is the collision time; $\kappa$ is given by $\kappa=6 \pi \varepsilon_{0} c_{3} / \omega_{l}^{2} \tau_{32} ; \Delta N=N_{2}-N_{3}$ is the population density difference between the populations in levels 2 and 3 .

For a TE field, the corresponding equations are

$$
\begin{aligned}
& \frac{\partial H_{z}}{\partial y}=\varepsilon_{0} \varepsilon_{i} \frac{\partial E_{x}}{\partial t}+\frac{\partial P_{x}}{\mathrm{~d} t}, \\
& -\frac{\partial H_{z}}{\partial x}=\varepsilon_{0} \varepsilon_{i} \frac{\partial E_{y}}{\partial t}+\frac{\partial P_{y}}{\mathrm{~d} t}, \\
& \frac{\partial E_{y}}{\partial x}-\frac{\partial E_{x}}{\partial y}=-\mu_{0} \frac{\partial H_{z}}{\partial t}, \\
& \frac{\mathrm{d} N_{1}}{\mathrm{~d} t}=\frac{N_{2}}{\tau_{21}}+\frac{N_{3}}{\tau_{31}}-N_{1} W_{p}, \\
& \frac{\mathrm{d} N_{2}}{\mathrm{~d} t}=\frac{N_{3}}{\tau_{32}}-\frac{N_{2}}{\tau_{21}}-\frac{N_{2}}{T}+\frac{\vec{E}}{\hbar \omega_{l}} \cdot \frac{\mathrm{d} \vec{P}}{\mathrm{~d} t}, \\
& \frac{\mathrm{d} N_{3}}{\mathrm{~d} t}=N_{1} W_{p}-\frac{N_{3}}{\tau_{31}}-\frac{N_{3}}{\tau_{32}}+\frac{N_{2}}{T}-\frac{\vec{E}}{\hbar \omega_{l}} \cdot \frac{\mathrm{d} \vec{P}}{\mathrm{~d} t}, \\
& \frac{\mathrm{d}^{2} \vec{P}}{\mathrm{~d} t^{2}}+\Delta \omega_{l} \frac{\mathrm{d} \vec{P}}{\mathrm{~d} t}+\omega_{l}^{2} \vec{P}=\kappa \Delta N \vec{E},
\end{aligned}
$$

where $\vec{E}=E_{x} e_{x}+E_{y} e_{y}$ and $\vec{P}=P_{x} e_{x}+P_{y} e_{y}$ for a TE field.

The values used for these parameters in that calculations are: $T_{2}=2 \times 10^{-14} \mathrm{~s}, \tau_{32}=1.1 \times 10^{-9} \mathrm{~s}, \tau_{21}=3 \times 10^{-3} \mathrm{~s}, \tau_{31}=$ $3 \times 10^{-6} \mathrm{~s}$ and $N_{\mathrm{T}}=\sum_{i=1}^{3} N_{i}=1.6 \times 10^{25} \mathrm{~m}^{3}$.

To excite the system, we must introduce sources [12]. Therefore, a broadband Gaussian pulse with a random am- 
plitude was used as an excitation source in our system. Then, using FDTD methods and Perfectly Matched Layer (PML) absorbing boundary condition, the electromagnetic fields can be calculated by solving the equations listed above. In the FDTD calculation, we discretize both space and time. The discrete space and time steps were chosen to be $\Delta x$ $=2 \times 10^{-6} \mathrm{~m}$ and $\Delta t \leqslant \Delta x /(2 c)$, where $\Delta t$ is taken to be $2 \times 10^{-15}$ $\mathrm{s}$. The pulse response was recorded over a time window of length $T_{\mathrm{w}}=5.2 \times 10^{-10} \mathrm{~s}$ at all nodes in the system. It was then Fourier transformed to obtain the intensity spectrum.

\section{Numerical results}

First, we calculated the spectral intensity for various pumping rates for the TM field (Figure 2). In each panel, two peaks are marked by their central wavelengths $\lambda_{\mathrm{M} 1}$ and $\lambda_{\mathrm{M} 2}$. Note that every peak denotes a localized mode that is supported by the disordered medium. Hereinafter the subscripts $\mathrm{M}$ and $\mathrm{E}$ denote the TM and TE cases, respectively. From Figure 2(a), it can be seen that there are many discrete and weak peaks in the spectrum at a pumping rate of $W_{p}=100$ $\mathrm{s}^{-1}$. All of these peaks are within one order of magnitude in intensity. When the pumping rate is increased, the mode $\lambda_{\mathrm{M} 1}$ dominates because it is amplified preferentially. Also, its spectral width becomes broader at $W_{p}=0.9 \times 10^{3} \mathrm{~s}^{-1}$ than those at lower pumping rates. With further pumping rate increases, the spectral width of mode $\lambda_{\mathrm{M} 1}$ begins to decrease, but its peak intensity increases (Figure 2(c)). Then, another mode $\lambda_{\mathrm{M} 2}$ is also excited at $W_{p}=5.0 \times 10^{4} \mathrm{~s}^{-1}$ (Figure 2(d)). Finally, the peak intensities and widths of two modes reach to their steady-state values when the pumping rate reaches its largest values (Figure 2(e)).

Figure 3 is the counterpart to Figure 2 for the TE case. It can be seen from this figure that the spectra intensity and width evolution of the TE state is similar to that of the TM case.

To obtain more information on the threshold gain behavior for both the TM and TE modes, numerical calculations were performed at different pumping rates. From these calculations we can obtain the curves of the peak intensity vs the pumping rate. This is shown in Figure 4. The selected modes have the minimum lasing threshold for both the TM and TE modes. The wavelength of the TM mode is $\lambda_{\mathrm{M} 1}=$ $332.8 \mu \mathrm{m}$, and the wavelength of the TE mode is $\lambda_{\mathrm{E} 1}=332.1$ $\mu \mathrm{m}$. Using a conventional method, the pump thresholds for the two modes can be measured from the intensity curves in Figure 4. For this calculation we obtained $W_{\mathrm{M}}=0.9 \times 10^{3}$ $\mathrm{s}^{-1}$ and $W_{\mathrm{E}}=4.5 \times 10^{3} \mathrm{~s}^{-1}$. This shows that the TM mode has a lower threshold than the TE mode. This conclusion is in accord with the experimental results presented by Ito of Shizuoka University in the optical band [12].

We provide a monochromatic pulse at $332.5 \mu \mathrm{m}$ to pump the medium at all sites to obtain the spatial distributions of both the TM and TE modes. Figure 5 shows that the two modes have different spatial distributions. The spatial distribution of the TM mode is much more centralized than that of the TE mode.
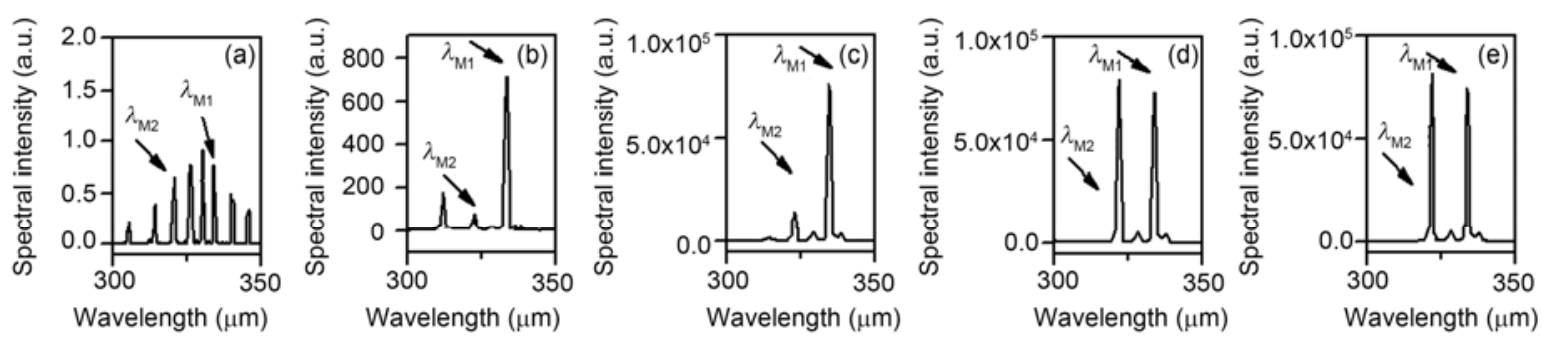

Figure 2 Spectral intensity vs. the wavelength $(\lambda)$ for TM fields in the $2 \mathrm{D}$ random medium. (a) $W_{p}=100 \mathrm{~s}^{-1} ;$ (b) $W_{p}=0.9 \times 10^{3} \mathrm{~s}^{-1} ;(\mathrm{c}) W_{p}=1.0 \times 10^{4} \mathrm{~s}^{-1} ;(\mathrm{d})$ $W_{p}=5.0 \times 10^{4} \mathrm{~s}^{-1} ;(\mathrm{e}) W_{p}=1.0 \times 10^{5} \mathrm{~s}^{-1}$.
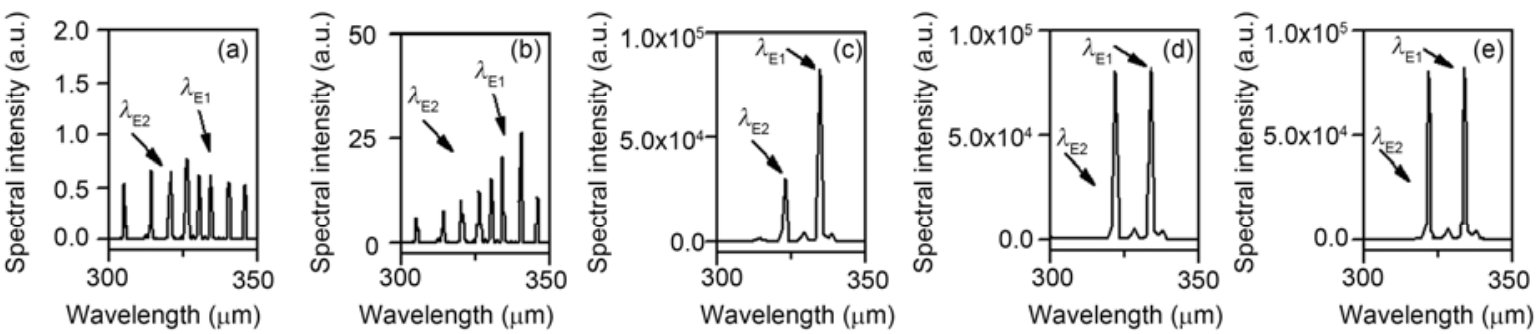

Figure 3 Spectral intensity vs. wavelength $(\lambda)$ for TE fields in the $2 \mathrm{D}$ random medium. (a) $W_{p}=100 \mathrm{~s}^{-1} ;$ (b) $W_{p}=0.9 \times 10^{3} \mathrm{~s}^{-1} ;(\mathrm{c}) W_{p}=1.0 \times 10^{4} \mathrm{~s}^{-1} ;(\mathrm{d}) W_{p}$ $=5.0 \times 10^{4} \mathrm{~s}^{-1}$; (e) $W_{p}=1.0 \times 10^{5} \mathrm{~s}^{-1}$. 


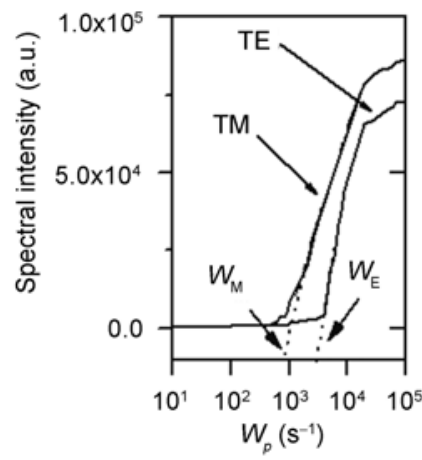

Figure 4 Peak intensity of the mode with the minimum lasing threshold vs. the pumping rate for both the TE and TM cases.
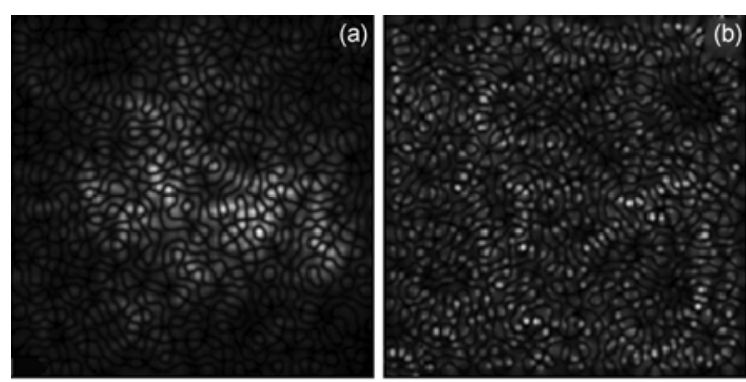

Figure 5 Spatial distribution of the field amplitude corresponding to the marked modes. (a) TM mode; (b) TE mode.

To determine whether or not the above results can be universally applied, we performed many calculations for different combinations of system parameters. All the calculated results demonstrate that our conclusion is universal.

\section{Conclusion}

In summary, a numerical study of a two-dimensional disordered media made of ruby grains with a three-level atomic system was performed. The calculation results show that $\mathrm{THz}$ random lasing can occur in a $2 \mathrm{D}$ disordered medium, and that the lasing threshold of TM fields is lower than that of TE fields.

This work was supported by the National Natural Science Foundation of China (60778003), the Fundamental Research Funds for the Central Universities (2010MS041) and the Science Foundation of China Academy of Enginnering Physics NSAF (10876010).

1 Letokhov V S. Generation of light a scattering medium with negative resonance absorption. Sov Phys JETP, 1968, 26: 835-840

2 Lawandy N M, Balachandra R M, Gomes A S L, et al. Laser action in strongly scattering media. Nature, 1994, 368: 436-438

3 Wiersma D S. The physics and applications of random lasers. Nat Phys, 2008, 4: 359-367

4 Cao H, Zhao Y G, Ong H C, et al. Random laser action in semiconductor powder. Phys Rev Lett, 1999, 82: 2278-2281

5 Cao H, Xu J Y, Zhang D Z, et al. Spatial confinement of laser light in active random media. Phys Rev Lett, 2000, 84: 5584-5587

6 Jiang X, Soukoulis C M. Time dependent theory for random lasers. Phys Rev Lett, 2000, 85: 70-73

7 Sebbah P, Vanneste C. Random laser in the localized regime. Phys Rev B, 2002, 66: 144202-144211

8 Jiang X, Soukoulis C M. Localized random lasing modes and a path for observing localization. Phys Rev E, 2002, 65: 025601-025604

9 Soukoulis C M, Jiang X, Xu J Y, et al. Dynamic response and relaxation oscillations in random lasers. Phys Rev B, 2002, 65: 041103-041106

10 Li Q M, Ho K M. Mode distribution in coherently amplifying random media. Physica B, 2001, 296: 78-84

11 Cao H, Jiang X Y, Ling Y, et al. Mode repulsion and mode coupling in random lasers. Phys Rev B, 2003, 67: 161101-161104

12 Ito T, Tomita M. Polarization-dependent laser action in a two-dimensional random medium. Phys Rev E, 2002, 66: 027601-027605

13 Wang C, Liu J S. Polarization dependence of lasing modes in two-dimensional random lasers. Phys Lett A, 2006, 353: 269-272

14 Liu J S, Xiong Z, Wang C. Theoretical investigation on polarization-dependent laser action in two-dimensional random media. J Opt A, 2007, 9: 658-663

15 Türeci H E, Ge L, Rotter S, et al. Strong interactions in multimode random lasers. Science, 2008, 320: 643-646

16 Vanneste C, Sebbah P, Cao H. Lasing with resonant feedback in weakly scattering Random systems. Phys Rev Lett, 2007, 98: 143902-143905

17 Liu J S, Liu Y, Lü J T, et al. Numerical study on terahertz random lasing in disordered ruby with three-level atomic system. Opt Exp, 2010, 18: 22880-22885

18 Yan Y, Liu S G, Li X Y, et al. Design and demonstration of a 0.22 THz gyrotron oscillator. Chinese Sci Bull, 2009, 54: 1495-1499

19 Hou B H, Wang Y L, W Y C, et al. THz spectra of five borates crystals. Chinese Sci Bull, 2008, 53: 155-158

20 Zhang C L, Zhao G Z. THz time-domain spectroscopy of amino acids. Chinese Sci Bull, 2005, 50: 1561-1565

21 Xu J Z, Wang L, Yang G Z. Effects of spectral linewidth of ultrashort pulses on the spatiotemporal distribution of diffraction fields. Chinese Sci Bull, 2001, 46: 901-904

22 Ge M, Zhao H W, Ji T, et al. Terahertz time-domain spectroscopy of some pentoses. Sci China Ser B-Chem, 2006, 49: 204-208

23 Yu R J, Zhang B. A new generation of plastic optical fibers and its functional exploiting. Sci China Ser E-Tech Sci, 2008, 51: 2207-2217

24 Ge M, Zhao H W, Wang W F, et al. Terahertz time-domain spectroscopic investigation on quinones. Sci China Ser B-Chem, 2008, 51: $354-358$

25 Zhang X C, Xu J Z. Introduction to THz Wave Photonics. Berlin: Springer, 2010

26 Lawandy N M. Far-infrared lasing in ruby. IEEE J Quant Electron, 1979, 6: 401-403

Open Access This article is distributed under the terms of the Creative Commons Attribution License which permits any use, distribution, and reproduction in any medium, provided the original author(s) and source are credited. 